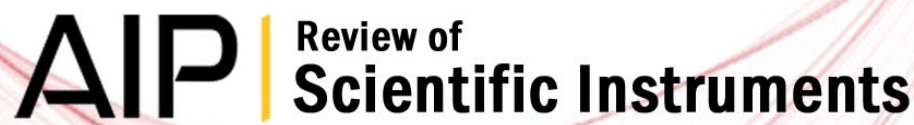

Note: Focus error detection device for thermal expansion-recovery microscopy (ThERM)

E. A. Domené and O. E. Martínez

Citation: Rev. Sci. Instrum. 84, 016104 (2013); doi: 10.1063/1.4774111

View online: http://dx.doi.org/10.1063/1.4774111

View Table of Contents: http://rsi.aip.org/resource/1/RSINAK/v84/i1

Published by the AIP Publishing LLC.

\section{Additional information on Rev. Sci. Instrum.}

Journal Homepage: http://rsi.aip.org

Journal Information: http://rsi.aip.org/about/about_the_journal

Top downloads: http://rsi.aip.org/features/most_downloaded

Information for Authors: http://rsi.aip.org/authors 


\title{
Note: Focus error detection device for thermal expansion-recovery microscopy (ThERM)
}

\author{
E. A. Domené ${ }^{1}$ and O. E. Martínez $z^{1,2, a)}$ \\ ${ }^{1}$ Laboratorio de Electrónica Cuántica (LEC), Departamento de Física, Facultad de Ciencias Exactas y \\ Naturales, Universidad de Buenos Aires (UBA), Buenos Aires, Argentina \\ ${ }^{2}$ Tolket SRL, Int. Güiraldes 2160, Pabellón I, Cdad. Universitaria, CABA C1428EAH, Buenos Aires, Argentina
}

(Received 5 September 2012; accepted 18 December 2012; published online 8 January 2013)

\begin{abstract}
An innovative focus error detection method is presented that is only sensitive to surface curvature variations, canceling both thermoreflectance and photodefelection effects. The detection scheme consists of an astigmatic probe laser and a four-quadrant detector. Nonlinear curve fitting of the defocusing signal allows the retrieval of a cutoff frequency, which only depends on the thermal diffusivity of the sample and the pump beam size. Therefore, a straightforward retrieval of the thermal diffusivity of the sample is possible with microscopic lateral resolution and high axial resolution $(\sim 100 \mathrm{pm})$. (c) 2013 American Institute of Physics. [http://dx.doi.org/10.1063/1.4774111]
\end{abstract}

There are in the literature many different approaches for measuring thermal properties in solids, in particular thermal diffusivity. Despite the wide range of techniques and approaches that exist, their use for retrieval of information at microscopic levels possesses inherent difficulties: IR techniques are diffraction limited ${ }^{1-4}$ thermoreflectance ${ }^{5-8}$ requires the independent scan of two beams to determine the phase delay as a function of the distance between pump and probe, or a very stringent requirement in the pointing stability if collinear beams are used and a frequency scan is performed; this same restriction applies to photodeflection techniques ${ }^{9-11}$ where the two beams must be kept at a constant separation.

In a recent paper, Mingolo ${ }^{12}$ has proposed a photothermal method based on sensing the focus shift of a probe beam produced by the curvature of the sample due to thermal expansion. This technique relies on measuring the signal reinjected into the optical fiber used to deliver both pump and probe laser beams (guarantees collinearity). The measured signal has contributions from both the focus shift and thermoreflectance. From fitting both phenomena, a value for the cutoff frequency is obtained and thus the thermal diffusivity of the sample.

In this work, we present a different experimental approach to characterize the surface curvature that has the advantage of cancelling the thermoreflectance contribution and that instead of requiring a focus shift to maximize the signal, the best configuration is precisely at focus. We call this method thermal expansion-recovery microscopy since we are sensitive to the change in thermal expansion at a microscopic level due to the thermal properties of the sample.

The expression for the temperature rise and thermal expansion of a sample due to heating can be obtained by solving the heat diffusion equation. ${ }^{13-15}$ The thermal expansion not only defocuses the probe beam due to the surface displacement but also due to the surface deformation (curvature). The inverse of the radius of curvature $(R)$ may be related to the

\footnotetext{
${ }^{a)}$ Member of the technical staff of CONICET.
}

thermal expansion and the pump beam size $\mathrm{e}^{12}$

$$
\frac{1}{R}=-\frac{P_{\omega}}{\sigma_{\text {pump }}^{2}} \frac{\lambda_{z} \eta}{4 \pi \kappa_{r}} \int_{0}^{\infty} \frac{e^{-u}}{u+i \omega / \omega_{0}} u d u
$$

where $\lambda_{z}$ is the linear expansion coefficient in the $\mathrm{z}$ direction, $\eta$ is the portion of incident light absorbed by the material, $P_{\omega}$ is the modulated pump laser power, $\kappa_{r}$ is the radial thermal conductivity and

$$
\omega_{0}=2 \pi \cdot f_{0}=2 D_{r} / \sigma_{\text {pump }}^{2}
$$

is the cutoff frequency, where $D_{r}$ is the desired thermal diffusivity and $\sigma_{\text {pump }}$ is the pump Gaussian beam size.

If we introduce a curvature at the focal plane of a Gaussian beam, the effect is a corresponding shift of the focal plane in a distance $\Delta f=R /\left(1+R^{2} / z_{0}^{2}\right)$, where $z_{0}$ is the confocal parameter of the beam. For small thermal expansions, we obtain a large $R$, which allows us to express the shift in the focal plane as a ratio to the confocal parameter: $\Delta f / z_{0} \underset{R \gg z_{0}}{\longrightarrow} z_{0} / R$.

The total focus shift has two contributions: one due to the movement of the reflecting surface caused by the thermal expansion; one due to the defocusing introduced by the presence of a curved surface. By calculating the ratio between the surface curvature focal plane shift and the thermal expansion $\left(\Delta f /\left.\delta L_{z}\right|_{\omega}\right)$ at the cutoff frequency $\left(\omega_{0}\right)$ for our particular case with a probe beam of $4 \mu \mathrm{m}$, we see that the curvature effect is about 3000 times stronger than the thermal expansion.

The experimental setup consists of a pump-probe scheme coupled to a commercial inverted microscope (Olympus IX71) through the left side camera port (see Figure 1). The pump is an amplitude modulated $784 \mathrm{~nm}$ laser diode; the probe is a CW $658 \mathrm{~nm}$ laser diode. To be able to perform 2D scans of the sample with a focused beam, a pair of galvo mirrors are located at the image plane of the back focal plane of the microscope objective. Each laser has its pair of alignment mirrors to be able to make both beams collinear. A collimator is placed at the output of the pump laser for two reasons: to compensate for differences in the divergence of both beams; change the size of the pump beam and thus modify the 


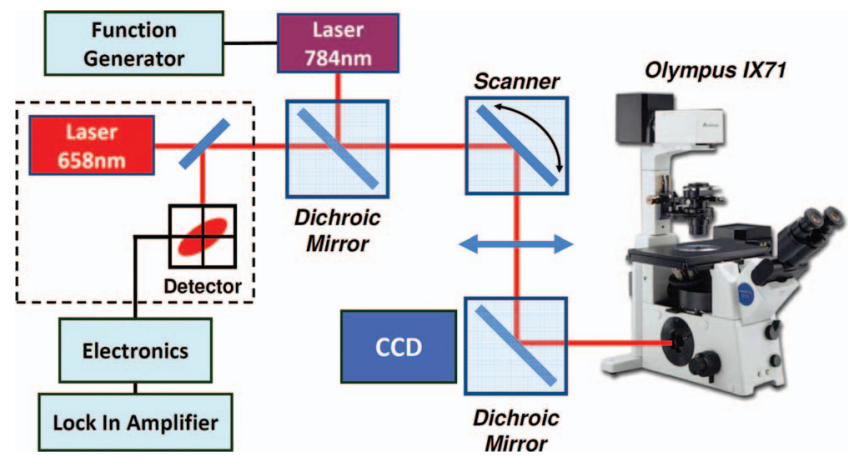

FIG. 1. Experimental setup: a pump-probe scheme with a four-quadrant detector coupled to an inverted commercial microscope.

cutoff frequency (Eq. (2)). The use of a $4 \mathrm{X}$ Olympus objective results in a pump beam area of around $16 \mu \mathrm{m}^{2}$.

The defocusing of the sample, caused by the heating due to absorption of the pump, is detected by monitoring a focus error (FE) signal generated from the four-quadrant detector inside a DVD pickup head which contains the probe laser. Commercial DVD pickups use an astigmatic beam which is reflected on the surface of the DVD and then impinges on a four-quadrant detector. ${ }^{16}$ The FE signal is calculated by subtracting the sums of each of the diagonals of the four-quadrant detector, resulting in a signal with a linear range, proportional to the defocusing of the probe beam. Sensitivity of the FE signal depends on the reflectivity of the sample, the probe laser power, the confocal parameter of the focused probe beam (choice of microscope objective), and the gain in the electronics that generates the error signal from the individual signals of the four-quadrant detector. A shortpass filter is placed before the probe laser pickup head in order to filter the pump laser from the four-quadrant detector.

The sample is then placed on the microscope and the FE signal is monitored by a digital oscilloscope to assure that there is no variation of the continuous component, which might be due to a mechanical drift in the microscope. In order to neglect undesired thermoreflectance ${ }^{9,17}$ contributions to the FE signal, the sample must be placed in the focal plane of the probe laser. This is done by moving the objective of the microscope until the FE signal is equal to $0 \mathrm{~V}$. In other words, the reflected probe beam balances the two diagonals of the four-quadrant detector and thus the FE signal is null. Since both diagonals have the same contribution from thermoreflectance, the subtraction performed to generate the FE signal eliminates the presence of thermoreflectance. But the CCD camera must also image the sample surface to measure the pump beam size. Both requirements can be met by moving the CCD camera.

Both pump and probe beam are made collinear to avoid the presence of deflection in the FE signal. The deflection signals (deflection in the $\mathrm{x}$ and $\mathrm{y}$ axis of the four-quadrant detector) can be monitored with the lock-in amplifier and minimized by aligning the beams. Numerical simulations of the analytical frequency dependent deflection signal ${ }^{9}$ were performed. Then the amplitude of these curves was fitted using the frequency dependence of Eq. (1): up to a $40 \%$ relative center offset of the pump and probe beam resulted in less than a $10 \%$ change in the cutoff frequency.

A lock-in amplifier is used to measure amplitude and phase of the modulated FE signal as a function of the frequency. In order to normalize the measured signal, accounting for phase shifts due to cables and electronics as well as compensate frequency related power variations of the pump laser, a zero-phase is measured: the shortpass filter is removed, the probe laser is turned off, and a frequency sweep is performed, where the measured FE signal corresponds to the reflected pump laser impinging on the four-quadrant detector; then, using the shortpass filter, a new frequency sweep of the FE signal is measured which quantifies the defocusing of the probe beam due to the surface curvature. Images of both pump and probe laser are taken with the CCD camera and later analyzed using a 2D Gaussian fit.

Measurements were taken on two different samples: polished bismuth $(\mathrm{Bi})$ and a microscope cover glass with a sputtered Pt film of $(4.5 \pm 0.3) \mathrm{nm}$. The thickness of the film was obtained from fitting transmission spectra ${ }^{18}$ with experimental data of the real and imaginary parts of the refractive index for a thin Pt film. ${ }^{19}$ The Pt film is used to increase both the reflectivity of the glass substrate at the probe wavelength, as well as the absorption at the pump wavelength, allowing the measurement of both transparent and opaque samples. The Pt absorption acts as a heat source for the glass substrate ${ }^{20}$ and due to the thickness of the sputtered film the sample is not analyzed by a two layer model; only the glass substrate is taken into account theoretically to model the defocusing.

To test this hypothesis, numerical simulations were performed. We analytically solved the thermal expansion and thus obtained the curvature signal for a single absorbing layer and then for a two layer model. The numerical results of the surface deformation due to curvature show that the presence of a Pt film has a stronger impact on the phase than on the amplitude of the signal: at the high end of the frequency range used in this experiment, the phase deviates noticeably from that of the single layer model; whilst the deviation of the amplitude is less noticeable. Therefore, with these results in mind, only the amplitude of the measured signal was used to perform the fitting of the experimental data.

Calculating the heat flux ratio ${ }^{20}$ between the Pt film and the glass substrate shows that the heat flux in the Pt represents only $7 \%$ of the total heat flux of the glass. We expect contributions from the Pt film to appear with a decrease in the pump beam size and an increase in the thickness of the film.

In Figure 2, we show the normalized measurements of frequency sweeps of the FE signals for both samples (square markers). Only the amplitude of the signal is shown and fitted. The corresponding nonlinear fits of the experimental points are plotted (full line). The insets show the 2D Gaussian fits of the pump beam at the sample plane, as well as a picture of the sample itself.

Both samples were measured using a pump power of $15.7 \mathrm{~mW}$ at the sample plane (less than $5 \mathrm{~mW}$ can be used). From the nonlinear fit, using the frequency dependence of Eq. (1), the cutoff frequency is obtained. The thermal diffusivity is then calculated using Eq. (2) and the pump beam size. For the polished Bi sample (Figure 2(a)), we obtain a thermal 

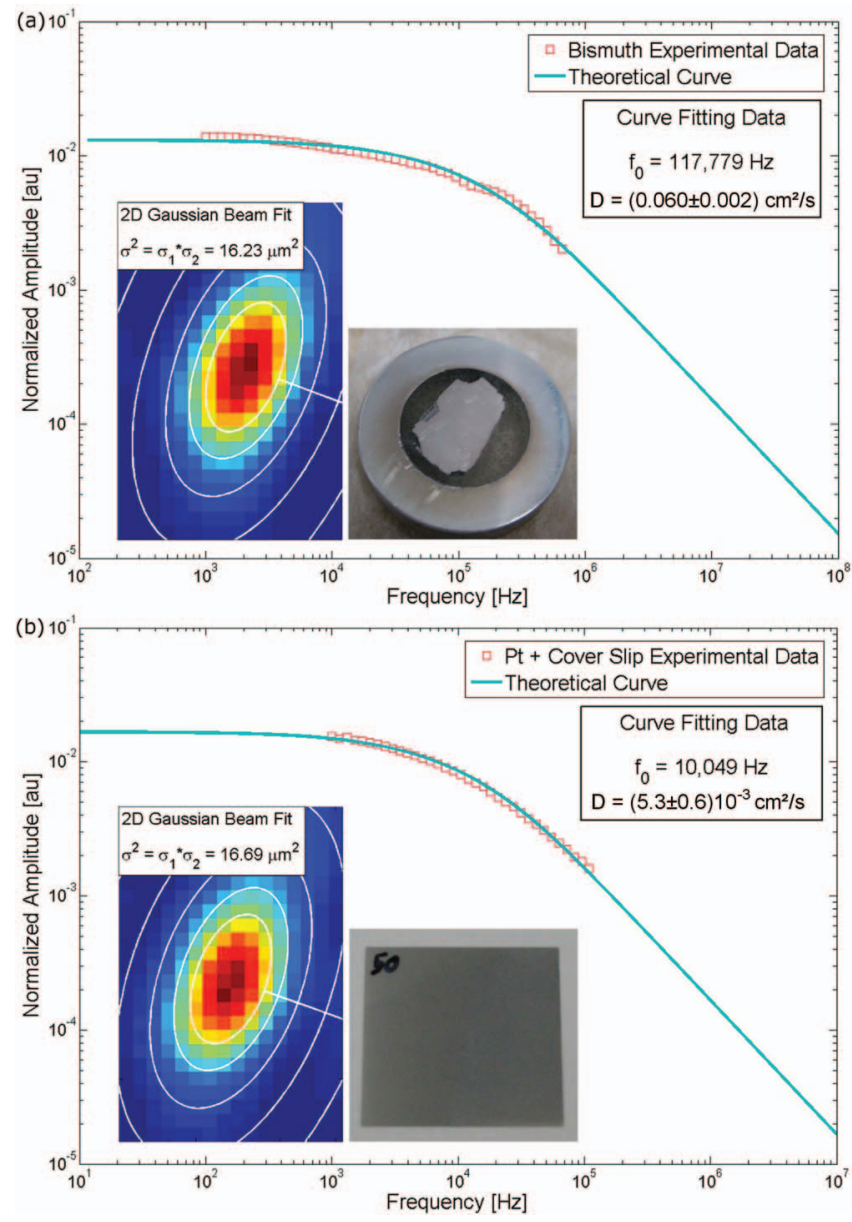

FIG. 2. Experimental frequency sweeps (square markers) and corresponding nonlinear fits (solid line) for (a) polished Bismuth and (b) Pt film on glass microscope cover slip. The insets of each graph show the 2D Gaussian fit for the pump beam and a picture of the measured sample.

diffusivity of $(0.060 \pm 0.002) \mathrm{cm}^{2} / \mathrm{s}$. The reference value for the Bi thermal diffusivity ${ }^{21}$ is $0.066 \mathrm{~cm}^{2} / \mathrm{s}$, which is very close to our measured value.

The polished Bi sample could be alternatively used as a standard for other samples by obtaining the pump beam size from the cutoff frequency and the reference value of the thermal diffusivity of $\mathrm{Bi}$ and thus no longer depending on the calibration of the CCD camera. By doing so, and using the cutoff frequency from the nonlinear fit in Figure 2(b), we obtain a thermal diffusivity of $(0.0056 \pm 0.0006) \mathrm{cm}^{2} / \mathrm{s}$ for the glass cover slip. On the other hand, if we use the same cutoff frequency from the nonlinear fit in Figure 2(b) together with the 2D Gaussian fit of the pump beam, we obtain a value of $(0.0053 \pm 0.0006) \mathrm{cm}^{2} / \mathrm{s}$, which corresponds to the thermal diffusivity of the glass cover slip. Both values are consistent with typical values of glass thermal diffusivity that vary between 0.0043 and $0.0089 \mathrm{~cm}^{2} / \mathrm{s}$.

In conclusion, we show a non-contact, non-destructive photothermal technique with high axial resolution $(\sim 100 \mathrm{pm}$, which corresponds to the noise level of the FE signal), a lateral resolution of $4 \mu \mathrm{m}$ (with a $4 \times$ objective), and high sensitivity $(\sim 50 \mathrm{mV} / \mu \mathrm{m})$ capable of measuring $2 \mathrm{D}$ thermal diffusivity maps. Heat from the pump must be absorbed in a thin layer smaller than the beam size, but transparent or low absorbing samples can also be studied by sputtering a thin layer of Pt. The defocusing due to heating from a modulated pump laser is quantified by the error signal of a DVD pickup head, which makes use of an astigmatic beam and a fourquadrant detector. Experimental results are shown for a polished Bi sample and a microscope cover glass. In both cases, the measured thermal diffusivity is in good accordance with the reference values.

The detection scheme presented here, solves several drawbacks of prior techniques: it does not have such stringent requirements in the pointing stability of the pump and probe beams as the thermoreflectance and photodeflection technique; the contribution from thermoreflectance is cancelled out in the four-quadrant detector, avoiding the need for fitting two mechanisms simultaneously. Thus, less parameters must be fitted, rendering the technique more reliable. This is particularly relevant when mapping the sample at constant modulation frequency ${ }^{10}$ as the thermal diffusivity can be retrieved from a single measurement if only one mechanism contributes to the signal.

This study is based on research supported by grants from the University of Buenos Aires and ANPCYT.

${ }^{1}$ T. C. Ma, M. Munidasa, and A. Mandelis, J. Appl. Phys. 71, 6029 (1992).

${ }^{2}$ M. Munidasa, F. Funak, and A. Mandelis, J. Appl. Phys. 83, 3495 (1998).

${ }^{3}$ R. Celorrio, E. Apiñaniz, A. Mendioroz, A. Salazar, and A. Mandelis, J. Appl. Phys. 107, 083519 (2010).

${ }^{4}$ C. Glorieux, R. Li Voti, J. Thoen, M. Bertolotti, and C. Sibilia, J. Appl. Phys. 85, 7059 (1999).

${ }^{5}$ J. F. Bisson and D. Fournier, High Temp. - High Press. 30, 205 (1998).

${ }^{6}$ Li Bincheng, J. P. Roger, L. Pottier, and D. Fournier, J. Appl. Phys. 86, 5314 (1999).

${ }^{7}$ D. Fournier, MRS Bull. 26, 465 (2001).

${ }^{8}$ D. Rochais, H. Le Houëdec, F. Enguehard, J. Jumel, and F. Lepoutre, J. Phys. D: Appl. Phys. 38, 1498 (2005).

${ }^{9}$ O. E. Martínez, F. Balzarotti, and N. Mingolo, Appl. Phys. B 90, 69-77 (2008).

${ }^{10}$ U. Crossa Archiopoli, N. Mingolo, and O. E. Martínez, J. Appl. Phys. 107, 023520 (2010).

${ }^{11}$ U. Crossa Archiopoli, N. Mingolo, and O. E. Martínez, Surf. Coat. Technol. 205, 3087 (2011).

${ }^{12}$ N. Mingolo and O. E. Martínez, J. Appl. Phys. 111, 123526 (2012).

${ }^{13}$ J. Opsal, A. Rosencwaig, and D. L. Willenborg, Appl. Opt. 22, 3169-3176 (1983).

${ }^{14}$ W. B. Jackson, N. M. Amer, A. C. Boccara, and D. Fournier, Appl. Opt. 20, 1333-1344 (1981).

${ }^{15}$ S. M. Landi, A. V. Bragas, J. A. Coy, and O. E. Martínez, Ultramicroscopy 77, 207-211 (1999).

${ }^{16}$ K. Fan, C. Chu, and J. Mou, Meas. Sci. Technol. 12, 2137 (2001).

${ }^{17}$ J. Opsal, M. W. Taylor, L. Smith, and A. Rosencwaig, J. Appl. Phys. 61, 240 (1987).

${ }^{18}$ I. Chambouleyron, J. M. Martínez, A. C. Moretti, and M. Mulato, Appl. Opt. 36, 8238-8247 (1997).

${ }^{19}$ L. Goddard, K. Wong, A. Garg, E. Behymer, G. Cole, and T. Bond, in Proceedings of the IEEE LEOS Annual Meeting (IEEE, 2008), pp. 569570.

${ }^{20}$ E. A. Domené, F. Balzarotti, A. V. Bragas, and O. E. Martínez, Opt. Lett. 34, 3797-3799 (2009).

${ }^{21}$ See http://www.lookchem.com/Periodic-Table/Bismuth/ for uses, characteristics and properties of elements in the periodic table. 